\title{
Reconstruction Using Local Flaps for Penoscrotal Defects after Ablation of Skin Lesions
}

\author{
Hyunwoo Kyung, Hyeokjae Kwon, Seung-Han Song, Sang-Ha Oh \\ Department of Plastic and Reconstructive Surgery, Chungnam National University School of Medicine, Daejeon, Korea
}

\begin{abstract}
Background: Penoscrotal defects are associated with a variety of conditions, including extramammary Paget disease, Fournier's gangrene, and skin cancer. Careful reconstruction of the penoscrotal region is vital for sexual and urological functions. We used a variety of methods to reconstruct penoscrotal defects and obtained satisfactory results.

Methods: We performed a retrospective chart review of 13 patients who underwent penoscrotal reconstruction from January 2012 to May 2016. All patients underwent radical debridement. The defect sizes ranged from 4 to $225 \mathrm{~cm}^{2}$ (mean $75.0 \mathrm{~cm}^{2}$ ). The reconstructive procedures were performed using split-thickness skin grafts (STSGs) and scrotal advancement, pedicled anterolateral thigh (ALT), and internal pudendal artery perforator (IPAP) flaps.

Results: All of the flaps survived, with no reports of total flap necrosis or congestion. The overall surgical complication rate was $23.1 \%$. A hematoma occurred in 1 patient and wound dehiscence occurred in 2 patients; the problems resolved after a minor surgical procedure.

Conclusion: The selection of an appropriate reconstruction method should be based on the size, location, and severity of the defect in addition to the availability of local tissue. A skin graft is recommended for defects of the penile shaft. A scrotal flap is appropriate for the reconstruction of small scrotal defects, whereas the pedicled ALT flap and IPAP flap are recommended for large defects. We have proposed a flowchart for the reconstruction of penoscrotal defects that is based on the characteristics of the defects and our clinical experience.
\end{abstract}

Keywords: Reconstruction, Penile, Scrotum, Flaps

\section{Introduction}

Penoscrotal defects are associated with a variety of conditions, including extramammary Paget disease (EMPD), Fournier's gangrene (FG), and skin cancer. Careful reconstruction of the penoscrotal region is vital for sexual and urological functions [1]. The aims of reconstruction are to assist wound healing and provide adequate function and acceptable appearance. However, reconstruction of penoscrotal defects is difficult because of the variety of skin colors and thicknesses, the presence of bacterial flora, difficulty in immobilization, and complicated contours [1]. Therefore, a variety of approaches must be used for reconstruction, which are based on the size, position, and severity of the defect and the availability of surrounding tissue.

We reconstructed penoscrotal defects using local advancement scrotal flaps, skin grafting, anterolateral thigh (ALT) flaps, and internal pudendal artery perforator (IPAP) flaps and obtained satisfactory results. The purpose of the study was to review and analyze the results of our patients who underwent reconstruction of penile and scrotal defects.

\section{Original Article}

Received: November 1, 2017 Revised: December 26, 2017 Accepted: January 24, 2018

\section{Corresponding author: Hyunwoo Kyung, M.D.}

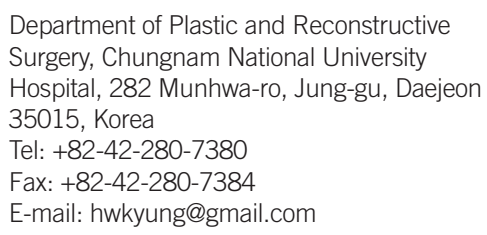

Department of Plastic and Reconstructive Surgery, Chungnam National University Hospital, 282 Munhwa-ro, Jung-gu, Daejeon 35015, Korea

Tel: $+82-42-280-7380$

Fax: +82-42-280-7384

E-mail: hwkyung@gmail.com

This is an Open Access article distributed under the terms of the Creative Commons Attribution Non-Commercial License (http://creativecommons.org/licenses/by-nc/4.0/) which permits unrestricted non-commercial use, distribution, and reproduction in any medium, provided the original work is properly cited.

(c) 2018 Korean Wound Management Society 


\section{Methods}

\section{Patients}

We performed a retrospective chart review of 13 patients who underwent penoscrotal reconstruction from January 2012 to May 2016. The defects were caused by EMPD in 10 patients, FG in 2 patients, and skin cancer in 1 patient. All patients underwent radical debridement. We applied a negative-pressure-wound-therapy (NPWT) device to facilitate wound healing and evacuation of discharges in selected patients who had a large wound defect and massive discharge. The defect areas ranged from 4 to $225 \mathrm{~cm}^{2}$ (mean $75.0 \mathrm{~cm}^{2}$ ). The reconstructive procedures were performed using split-thickness skin grafts (STSGs) and scrotal advancement, ALT, and IPAP flaps. The patients' demographic and clinical data are listed in Table 1.

\section{Surgical procedures}

Pedicled ALT flap

A line was drawn that connected the anterior superior iliac spine to the outer edge of the patella. The midpoint of the connection line was used to establish a circle with a radius of $3 \mathrm{~cm}$. We then used a portable Doppler ultrasound device to identify the pedicle skin perforators of the lateral femoral vessels within the circle. The flap was then mapped based on these vessels. The flap was elevated and the vasculature was anatomized to an appropriate length to ensure that the flap had sufficient mobility. A tunnel was made between the donor area and the defect, and the flap was transposed to the defect. If the width of the flap was less than $7 \mathrm{~cm}$, the donor area was usually sutured directly. The donor area was repaired by STSGs from the other thigh for flaps wider than $7 \mathrm{~cm}$.

\section{IPAP flap}

The gluteal fold was identified before surgery and demarcated bilaterally with the patient standing. Portable Doppler ultrasonography was used to locate perforators. The design of the pedicled flap was based on the marked perforators. To disguise the closure scar on the donor site, the long axis of the flap was centered on the gluteal fold. An exploratory skin

Table 1. Demographic and clinical data of the patients

\begin{tabular}{|c|c|c|c|c|c|c|c|}
\hline Case & Age & Diagnosis & Location & Defect size $(\mathrm{cm})$ & Reconstruction method & Complications & Follow up (mo) \\
\hline 1 & 75 & EMPD & Scrotum & $12 \times 7$ & Scrotal flap & Hematoma & 36 \\
\hline \multirow[t]{3}{*}{2} & 74 & EMPD & Pubic & $18 \times 10$ & ALT & None & 31 \\
\hline & & & Scrotum & $5 \times 7$ & Scrotal flap & & \\
\hline & & & Penile shaft & $8 \times 7$ & STSG & & \\
\hline 3 & 75 & FG & Scrotum & $5 \times 3$ & Scrotal flap & None & 24 \\
\hline \multirow[t]{2}{*}{4} & 85 & EMPD & Scrotum & $10 \times 5$ & Scrotal flap & None & 15 \\
\hline & & & Pubic & $5 \times 4$ & STSG & & \\
\hline \multirow[t]{2}{*}{5} & 68 & EMPD & Scrotum & $7 \times 3$ & Scrotal flap & None & 19 \\
\hline & & & Penile shaft & $6 \times 5$ & STSG & & \\
\hline 6 & 53 & EMPD & Scrotum & $10 \times 8$ & IPAP flap & Dehiscence & 18 \\
\hline 7 & 55 & EMPD & Penile shaft & $2 \times 2$ & Scrotal flap & None & 18 \\
\hline 8 & 53 & EMPD & Scrotum, pubic & $10 \times 7$ & Scrotal flap & None & 18 \\
\hline 9 & 81 & EMPD & Scrotum & $7 \times 5$ & STSG & None & 13 \\
\hline \multirow[t]{2}{*}{10} & 93 & EMPD & Penile shaft & $8 \times 7$ & STSG & None & 12 \\
\hline & & & Scrotum, pubic & $11 \times 10$ & ALT & & \\
\hline \multirow[t]{2}{*}{11} & 46 & EMPD & Penile shaft & $8 \times 7$ & STSG & Dehiscence & 12 \\
\hline & & & Scrotum & $17 \times 10$ & ALT & & \\
\hline \multirow[t]{2}{*}{12} & 54 & SCC & Penile shaft & $8 \times 7$ & STSG & None & 12 \\
\hline & & & Scrotum & $15 \times 15$ & IPAP flap & & \\
\hline \multirow[t]{2}{*}{13} & 65 & $F G$ & Penile shaft & $7 \times 6$ & STSG & None & 12 \\
\hline & & & Scrotum & $20 \times 8$ & IPAP flap & & \\
\hline
\end{tabular}

EMPD, extramammary Paget disease; FG, Fournier's gangrene; SCC, squamous cell carcinoma; STSG, split-thickness skin graft; ALT, anterolateral thigh; IPAP, internal pudendal artery perforator. 
incision was made on the anterior or posterior side of the flap, and either a suprafascial or subfascial dissection was performed until the previously marked perforators emerged. A final single perforator was selected after considering its pulsatility, diameter, and proximity to the defect. The remaining incision was made, and the perforator was freed from the connective tissue. The flap was transferred to the defect site, and a negative-suction drain was inserted. Donor sites were primarily secured within the gluteal folds.

\section{Results}

The ages of patients ranged from 46 to 93 years (mean $67.5 \pm 14.5$ years). The duration of follow-up ranged from 12 to 36 months (mean 18.5 months). Eight cases had a defect of the penile shaft that was repaired by an STSG. Seven cases who had no tension when the scrotal defect was approximated underwent repair using a scrotal advancement flap. A distant flap was performed for large scrotal defects that involved the surrounding tissue; 3 cases underwent repair by a pedicled ALT flap, and 3 patients underwent repair by an IPAP flap. The lengths of the flaps ranged from 2 to 20 $\mathrm{cm}$ (mean $9.5 \mathrm{~cm}$ ), and the widths ranged from 2 to $15 \mathrm{~cm}$ (mean $6.9 \mathrm{~cm}$ ). All of the flaps survived without reports of severe complications such as total flap necrosis or congestion that required immediate surgery. Minor complications (hematoma or wound dehiscence) occurred in 3 patients, which healed after a minor surgical procedure.

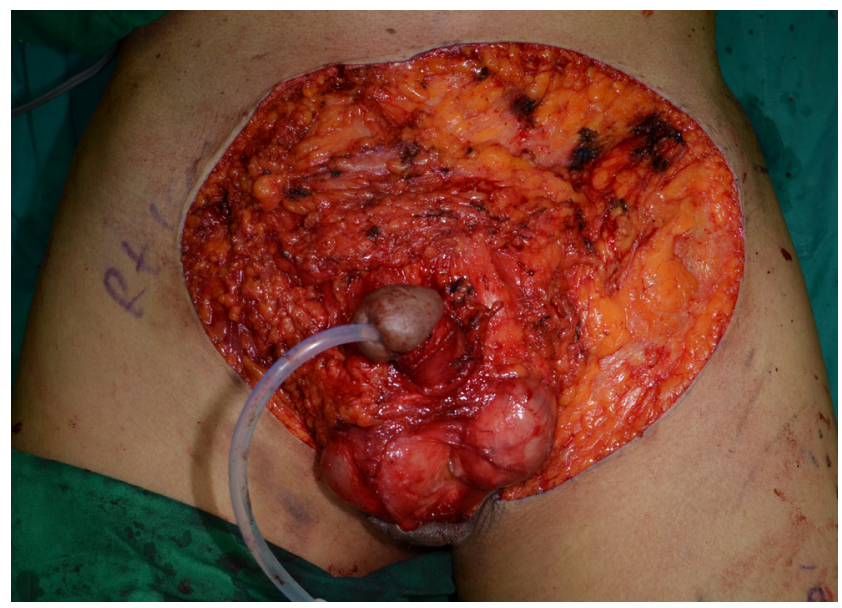

Fig. 1. (Case \# 2) A 74-year-old man with confirmed extramammary Paget disease. After complete excision, the areas of the penile, scrotal and pubic region defects were $56 \mathrm{~cm}^{2}(8 \times 7 \mathrm{~cm}), 35$ $\mathrm{cm}^{2}(5 \times 7 \mathrm{~cm})$, and $180 \mathrm{~cm}^{2}(18 \times 10 \mathrm{~cm})$, respectively.

\section{Case presentations}

Case 2

A 74-year-old man presented with a 2-year history of a pruritic erythematous skin lesion on his penis and a scrotal skin lesion. A skin biopsy confirmed the diagnosis of EMPD. After complete excision of the lesions, the resulting penile 56$\mathrm{cm}^{2}(8 \times 7 \mathrm{~cm})$, scrotal $35-\mathrm{cm}^{2}(5 \times 7 \mathrm{~cm})$, and pubic-region $180-\mathrm{cm}^{2}(18 \times 10 \mathrm{~cm})$ defects were reconstructed by an STSG, scrotal flap, and pedicled ALT flap, respectively (Figs. 1, 2). At the 31-month follow-up visit, local recurrence of EMPD was not seen, and the aesthetic result was acceptable (Fig. 3).

\section{Case 13}

A 65-year-old man sustained FG, with soft tissue defects over the penile shaft and scrotum. After surgical debridement, the areas of the defects were approximately $42 \mathrm{~cm}^{2}(7 \times 6 \mathrm{~cm})$ and $160 \mathrm{~cm}^{2}(20 \times 8 \mathrm{~cm})$, respectively. An IPAP flap was constructed and transposed to the scrotal defect. The penile shaft defect was repaired by an STSG (Figs. 4, 5). At postoperative month 12 , the flap had survived, and the aesthetic outcome was good (Fig. 6).

\section{Discussion}

The penoscrotal area has a special function that represents the confluence of urological, sexual, and gastrointestinal functions [1]. The scrotum allows testicular mobility, which reduces trauma and allows optimal thermal regulation for spermatogenesis [2]. The scrotum is constructed of special-

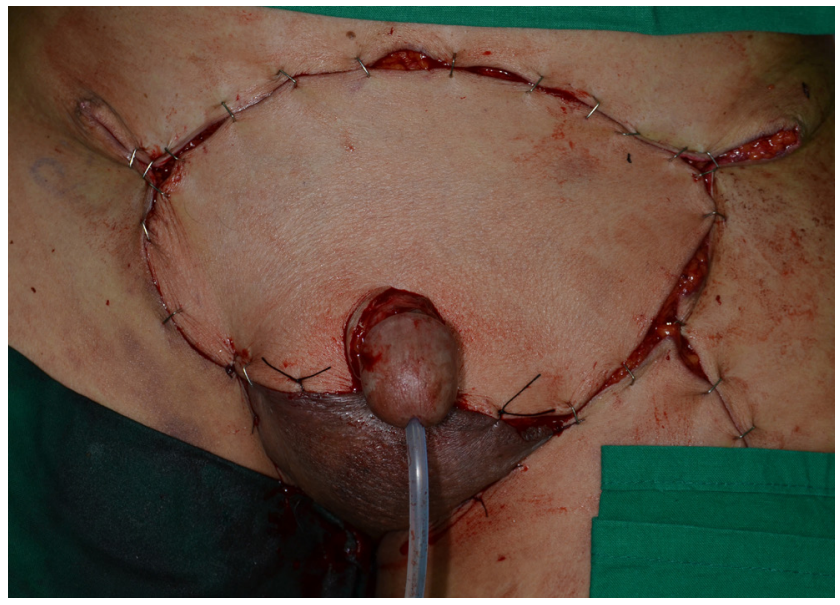

Fig. 2. (Case \# 2) A split-thickness skin graft and scrotal flap were used to repair the penile shaft and scrotal defect. An anterolateral thigh flap was elevated from the left thigh and was transposed to the defect in the pubic region. 


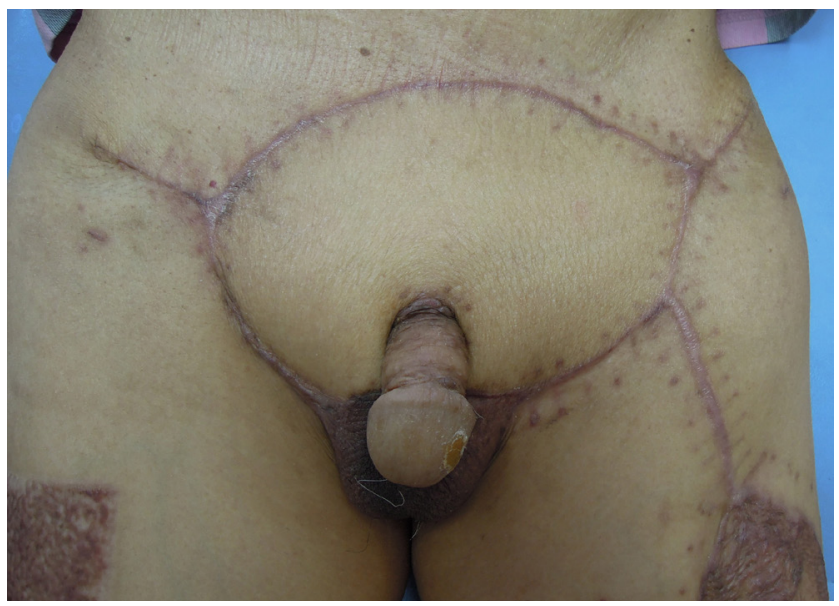

Fig. 3. (Case \# 2) Six months after surgery. The penile, scrotal, and pubic defects were completely covered and healed.

ized skin that is lined by the dartos muscle, which can contract to retain heat and relax to dissipate heat [2].

Penoscrotal defects may result from trauma, infection, or tumor ablation. FG is one of the most devastating types of infections. FG is a necrotizing fasciitis of the perineum and external genitalia that rapidly spreads along contiguous fascia. It often affects patients with serious comorbidities, including diabetes, alcoholism, and advanced age. In addition to cardiopulmonary support and broad-spectrum antibiotics, the standard treatment involves aggressive debridement of necrotic tissue. Consequently, reconstructive procedures are required for the resulting extensive skin and soft tissue defects [1].

EMPD is a rare cutaneous malignancy, and in men most frequently involves the penoscrotal area [3]. Defects due to tumor ablation are usually reconstructed immediately after the tumor is resected and the margin is confirmed free of malignancy. Wide excision and examination of frozen sections are needed to create and confirm tumor-free margins before reconstruction [3]. This type of surgery frequently leads to skin and soft tissue defects that are larger than expected.

Reconstruction of the penoscrotal region represents a surgical challenge because of the highly complex three-dimensional structures and the difficulties involved in achieving an esthetic appearance and normal functions. However, several reconstructive procedures can achieve wound coverage, including those using STSGs, local scrotal advancement flaps, and fasciocutaneous flaps [4].

Carvalho et al. [5] reported on a small defect of the scrotum that healed by secondary intention without surgical treat-

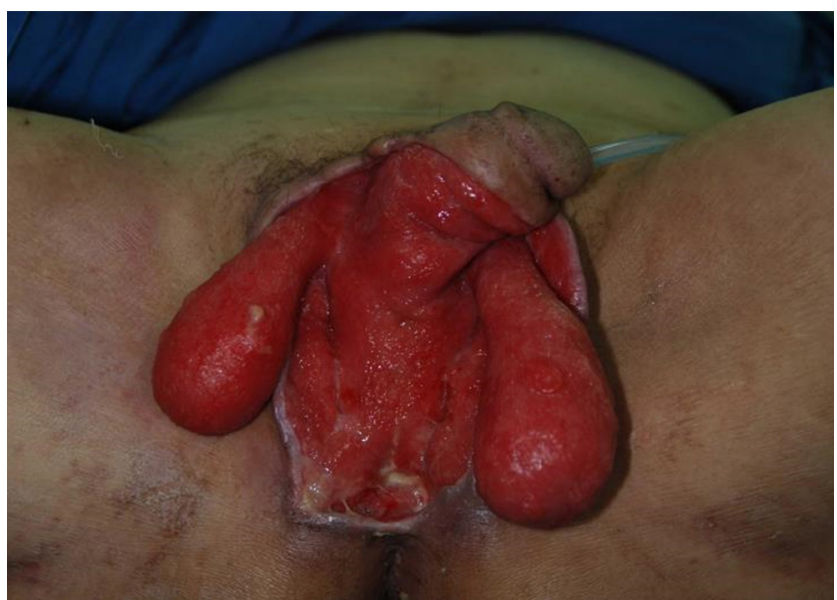

Fig. 4. (Case \# 13) A 65-year-old man sustained Fournier's gangrene with soft tissue defects over the penile shaft and scrotum. After surgical debridement, areas of the defects were approximately $42 \mathrm{~cm}^{2}(7 \times 6 \mathrm{~cm})$ and $160 \mathrm{~cm}^{2}(20 \times 8 \mathrm{~cm})$, respectively.

ment. Parkash et al. [6] reported good results after a skin graft, but Carvalho et al. [5] reported that infection occurred in $18 \%$ and Chen et al. [4] reported that partial skin loss occurred in $11 \%$ of skin grafts. The reason for the higher rates of infection or skin loss after skin grafting in the penoscrotal region compared with other sites is probably accounted for by the difficulties in immobilization, the presence of bacterial flora, and the complicated structures [1]. For our patients, STSGs were only used to cover penile shaft defects.

Parkash et al. [6] reported a successful reconstruction that was achieved by a local scrotal advancement flap and reported that wound dehiscence and partial flap loss occurred as complications but resolved, with flap survival. The authors also used a scrotal flap for reconstruction if the scrotal defect could be approximated without tension. However, another type of reconstructive surgery is needed if the defect is large and the remaining scrotal skin cannot be advanced and the surrounding defect is accompanied.

A fasciocutaneous flap provides durable skin and allows coverage of a large area of exposed soft tissue. Therefore, it is more effective than an STSG for the reconstruction of scrotal and perineal defects. It also produces better functional outcomes and cosmetic results than skin grafting, with fewer contractures due to scarring [1]. We used pedicle ALT flaps in 6 patients and IPAP flaps in 3 patients. Both flaps have advantages in that they are relatively easy to construct, have a reliable blood supply, and are less bulky than other muscle flaps. In most cases, the donor site allowed primary closure. However, when the width of the ALT flap was large, a skin 
Table 2. Studies reporting on reconstructive methods for penoscrotal defects

\begin{tabular}{lcclc}
\hline Author & Case number & Defect size $\left(\mathrm{cm}^{2}\right)$ & \multicolumn{1}{c}{ Reconstruction methods } & Complication rate (n) \\
\hline Chen et al. [4] & 31 & 86 & Scrotal flap, ALT, gracilis, STSG & $16 \%(5)$ \\
Ahn et al. [1] & 20 & 104.6 & LD free flap, ALT, STSG, perforator-based flap & $10 \%(3)$ \\
Chen et al. [9] & 5 & 90.5 & STSG, scrotal flap, gracilis, pudendal thigh flap & $40 \%(2)$ \\
Han et al. [12] & 10 & 151.7 & Scrotal flap, IPAP, STSG & $10 \%(1)$ \\
Lin et al. [13] & 10 & 122.5 & ALT & $10 \%(1)$ \\
Yao et al. [11] & 3 & 171.0 & ALT & $0 \%(0)$ \\
Yang et al. [8] & 5 & 160 & Rotary mutiflap & $0 \%(0)$ \\
Lee et al. [7] & 9 & 38.1 & Scrotal flap, groin flap, ALT & $0 \%(0)$ \\
Qin et al. [10] & 5 & 72 & Scrotal flap & $0 \%(0)$ \\
Our study & 16 & 90.4 & STSG, scrotal flap, ALT, IPAP & $25 \%(4)$ \\
\hline
\end{tabular}

STSG, split-thickness skin graft; LD, latissimus dorsi; EMPD, extramammary Paget disease; ALT, anterolateral thigh; IPAP, internal pudendal artery perforator.

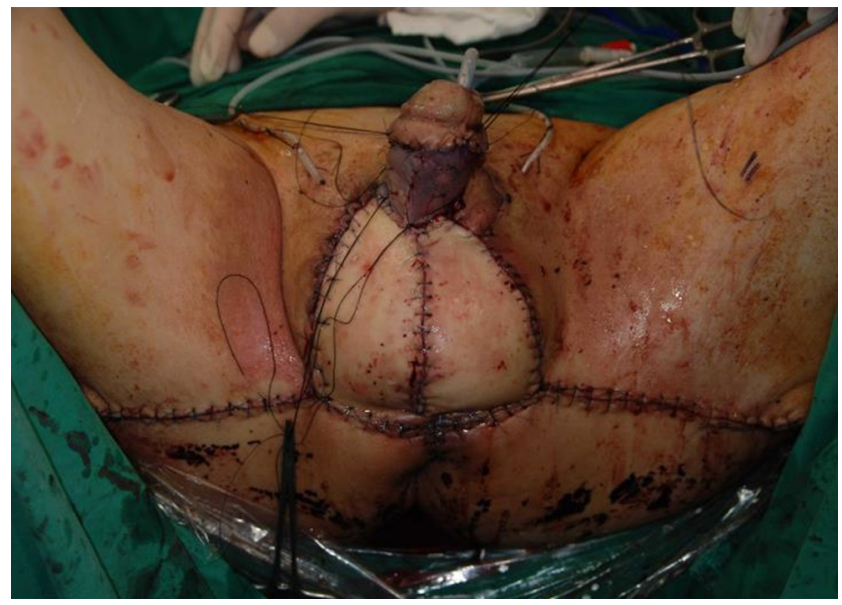

Fig. 5. (Case \# 13) An internal pudendal artery perforator flap was constructed and transposed to the defect. The defect on the penile shaft was covered by a split-thickness skin graft.

graft was performed for closure of the donor site. In addition, the construction of an ALT flap elevates a larger skin paddle than the IPAP flap, and a unilateral ALT flap can cover both exposed testicles. An ALT flap can be moved farther to cover the pubic area, so bilateral ALT flaps can be used for penoscrotal defects accompanied by defects in the pubic area. The IPAP flap should be used in a bilateral pattern when both testicles are exposed. For such cases, donor site scars are well concealed by both gluteal folds.

Table 2 summarizes our review of recent publications that have reported on reconstructive methods for defects of the penoscrotal area, and compares our study with the other case series. In 2007, Lee et al. [7] reported on reconstructions of penoscrotal defects by scrotal flaps, groin flaps, and pedicled ALT flaps. Yang et al. [8] reconstructed penoscrotal

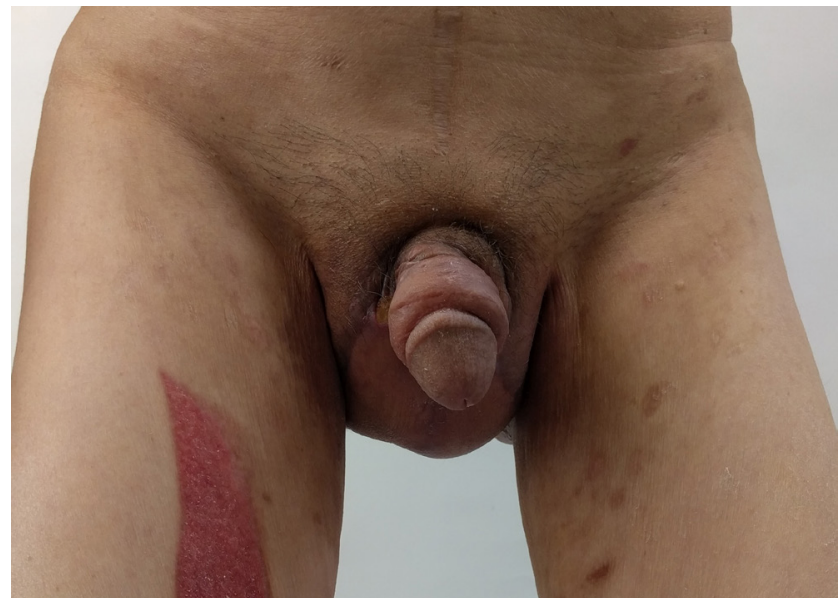

Fig. 6. (Case \# 13) Six months after reconstructive surgery, the scrotal and penile defect were completely covered and healed.

defects using superficial epigastric artery-based flaps and scrotal flaps. In 2010, Chen et al. [4] used gracilis muscle flaps and STSGs. In 2011, Chen et al. [9] reconstructed penoscrotal defects using conventional methods and pudendal thigh flaps. In 2014, Ahn et al. [1] performed reconstruction using LD free flaps and perforator-based flaps (IPAP flap) to repair penoscrotal defects. In addition, several published papers have reported on reconstruction of penoscrotal defects by the methods described so far. Currently, it appears that there is no general consensus on the methods used for reconstruction of penoscrotal defects. Thus, we recommend that the selection of an appropriate surgical procedure should be based on the location and size of the defect. Our flowchart for deciding on the appropriate procedure is shown in Fig. 7. 


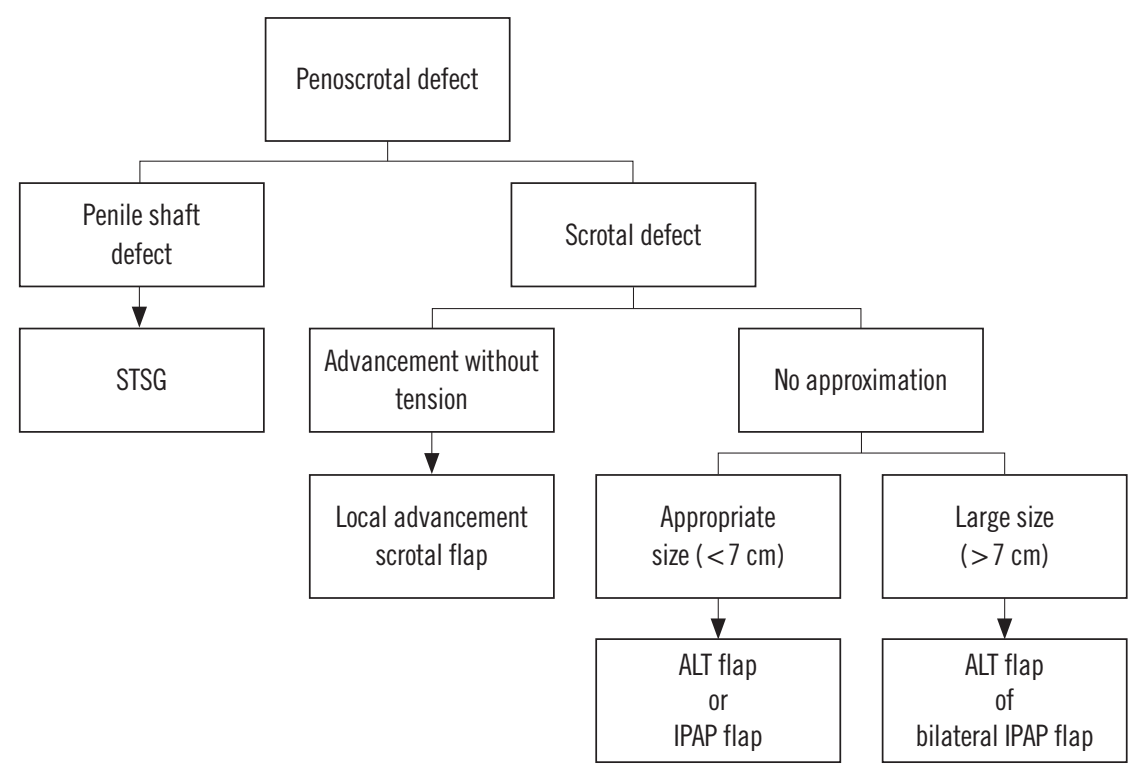

Fig. 7. Flowchart for reconstructing penoscrotal defects. STSG, split-thickness skin graft; ALT, anterolateral thigh; IPAP, internal pudendal artery perforator.

\section{Conclusions}

The appropriate reconstruction of a penoscrotal defect is vital for function and cosmesis. We used STSGs to repair defects on the penile shaft and local scrotal advancement flaps to repair relatively small scrotal defects. A distant flap was used for large defects that involved the surrounding tissue. Among distant flaps, the pedicled ALT flap can be used to reconstruct defects that expose both testicles, or the ALT flap can be mobilized to repair defects in the pubic area. An IPAP flap allows the donor site to be disguised within a gluteal fold. The authors suggest therefore, that a suitable approach for reconstruction of a penoscrotal defect is based on the size and position of the defect, and should involve a skin graft, scrotal flap, ALT flap, or IPAP flap.

\section{Conflict of interest}

No potential conflict of interest relevant to this article was reported.

\section{Acknowledgment}

The authors have certified that the process of the research is in accordance with ethical standards of Helsinki declaration, domestic and foreign committees that preside over human experiment.

\section{References}

1. Ahn DK, Kim SW, Park SY, et al. Reconstructive strategy and classification of penoscrotal defects. Urology 2014;84:121722.

2. Tran NV. Scrotal and perineal reconstruction. Semin Plast Surg 2011;25:213-20.

3. Lai YL, Yang WG, Tsay PK, et al. Penoscrotal extramammary Paget's disease: a review of 33 cases in a 20-year experience. Plast Reconstr Surg 2003;112:1017-23.

4. Chen SY, Fu JP, Chen TM, et al. Reconstruction of scrotal and perineal defects in Fournier's gangrene. J Plast Reconstr Aesthet Surg 2011;64:528-34.

5. Carvalho JP, Hazan A, Cavalcanti AG, et al. Relation between the area affected by Fournier's gangrene and the type of reconstructive surgery used. A study with 80 patients. Int Braz J Urol 2007;33:510-4.

6. Parkash S, Gajendran V. Surgical reconstruction of the sequelae of penile and scrotal gangrene: a plea for simplicity. Br J Plast Surg 1984;37:354-7.

7. Lee SR, Kang NH, Oh SH. Resurfacing the large penoscrotal defects with various local flaps after ablation of extramammary Paget's disease. J Korean Soc Plast Reconstr Surg 2007; 34:753-6.

8. Yang Q, Yu L, Wang L, et al. Rotary mutiflaps for defect reconstruction in penoscrotal extramammary Paget's disease. Urol Oncol 2008;26:600-3. 
9. Chen SY, Fu JP, Wang CH, et al. Fournier gangrene: a review of 41 patients and strategies for reconstruction. Ann Plast Surg 2010;64:765-9.

10. Qin X, Zhang S, Zhang H, et al. Reconstruction with scrotal skin flaps after wide local resection of penoscrotal extramammary Paget's disease. BJU Int 2012;110:E1121-4.

11. Yao H, Zheng D, Wen J, et al. Reconstruction of major scrotal defects by anterolateral thigh flap. Cell Biochem Biophys 2014;70:1331-5.
12. Han SE, Kim EJ, Sung HH, et al. Aesthetic Penoscrotal Resurfacing: Creating Propeller Flaps from Gluteal Folds. Eur Urol 2016 Oct 12 [Epub]. http://dx.doi.org/10.1016/j.eururo. 2016.09.033.

13. Lin CT, Chang SC, Chen SG, et al. Reconstruction of perineoscrotal defects in Fournier's gangrene with pedicle anterolateral thigh perforator flap. ANZ J Surg 2016;86:10525. 